\title{
Hidden Tiger: An Atypical Presentation of Anti-Glomerular Basement Membrane Disease
}

\author{
Ashani Lecamwasamª, Darren Lee ${ }^{\mathrm{a}, \mathrm{b}, \mathrm{d}}$, Alison Skene ${ }^{\mathrm{c}}$, Lawrence McMahon ${ }^{\mathrm{a}}$
}

\begin{abstract}
Anti-glomerular basement membrane (anti-GBM) disease is characterized by linear deposition of immunoglobulin $\mathrm{G}$ ( $\mathrm{IgG}$ ) along the GBM. The concurrence of anti-GBM disease with another type of glomerulonephritis has been well described. We report a case of necrotizing glomerulonephritis in association with prodromal systemic symptoms, deterioration in kidney function less rapid than expected, and the presence of circulating anti-GBM antibody. This was initially diagnosed histologically as immunoglobulin A (IgA) nephropathy based on immunohistochemistry (IHC) staining, but immunofluorescence (IF) staining on a subsequent biopsy was more consistent with a diagnosis of anti-GBM disease with coexisting mild IgA nephropathy. Prompt initiation of standard treatment of anti-GBM disease normalized kidney function. This case highlights that anti-GBM disease can present atypically and may also co-exist with another form of glomerulonephritis.
\end{abstract}

Keywords: Anti-glomerular basement membrane disease; Atypical; Immunofluorescence; Immunohistochemistry; Immunoglobu$\operatorname{lin} \mathrm{A}$

\section{Introduction}

Anti-glomerular basement membrane (GBM) disease is a rare condition characterized by the development of circulating autoantibodies against the alpha-3 chain of type IV collagen (alpha-3(IV)) of the GBM, the so-called Goodpasture autoantigen [1], resulting in rapidly progressive glomeru-

\footnotetext{
Manuscript accepted for publication July 21, 2014

a Department of Renal Medicine, Eastern Health Clinical School, Faculty of Medicine, Nursing \& Health Sciences Monash University, Victoria, Australia

${ }^{\mathrm{b}}$ Department of Nephrology, Austin Health, Victoria, Australia ${ }^{c}$ Department of Anatomical Pathology, Austin Health, Victoria, Australia ${ }^{\mathrm{d} C}$ Corresponding Author: Darren Lee, Department of Renal Medicine, Eastern Health, 2/F 5 Arnold Street, Box Hill, Victoria 3128, Australia. Email: Darren.Lee2@easternhealth.org.au
}

doi: http://dx.doi.org/10.14740/wjnu173w lonephritis (RPGN) [2]. When associated with pulmonary hemorrhage, it is referred to as Goodpasture's syndrome. The hallmark pathological features are extensive crescent formation and linear immunoglobulin $\mathrm{G}$ ( $\mathrm{IgG}$ ) deposition along the glomerular capillaries.

We describe a case of anti-GBM disease which lacked both the hallmarks of extensive crescents and linear IgG deposits by immunohistochemistry (IHC) staining on the initial and repeat biopsies, while the immunofluorescence (IF) staining on repeat biopsy demonstrated moderate but positive linear IgG staining, suggesting a possible difference in the sensitivity between IHC and IF methods for the detection of linear IgG deposition. Co-existing immunoglobulin A (IgA) nephropathy, as evident by mesangial IgA deposits and mild mesangial proliferation, less extensive crescent formation, as well as the subacute deterioration in kidney function, all confounded the initial diagnosis.

\section{Case Report}

A 56-year-old Caucasian man was referred to the Nephrology Outpatient Clinic with prodromal symptoms of lethargy, anorexia and myalgia for a month, associated with acute kidney injury, microscopic hematuria and proteinuria. $\mathrm{He}$ denied any respiratory symptoms (in particular hemoptysis), macroscopic hematuria, rash or arthralgia. His past medical history included a corneal transplant for keratoconus in childhood, eczema and hiatus hernia. Physical examination revealed a blood pressure of 135/80 mm Hg. He was clinically euvolemic without synovitis or rash.

Investigations revealed deterioration in his kidney function, with serum creatinine rising from $0.98 \mathrm{mg} / \mathrm{dL} 14$ months earlier, to $1.52 \mathrm{mg} / \mathrm{dL} 3$ weeks prior to his presentation, and $2.19 \mathrm{mg} / \mathrm{dL}$ on presentation. Urine microscopy showed $>1,000 \times 10^{6} / \mathrm{L}$ red cells without red cell casts, and spot urine protein-to-creatinine ratio was $0.50 \mathrm{mg} / \mathrm{g}$. Inflammatory markers were significantly elevated with an ESR of $106 \mathrm{~mm} / \mathrm{h}(<10)$ and CRP of $60 \mathrm{mg} / \mathrm{L}(<5)$. Antinuclear antibody (ANA), anti-neutrophil cytoplasmic antibody (ANCA) and cryoglobulin were all negative. Serum complement, rheumatoid factor, creatine kinase, and serum 

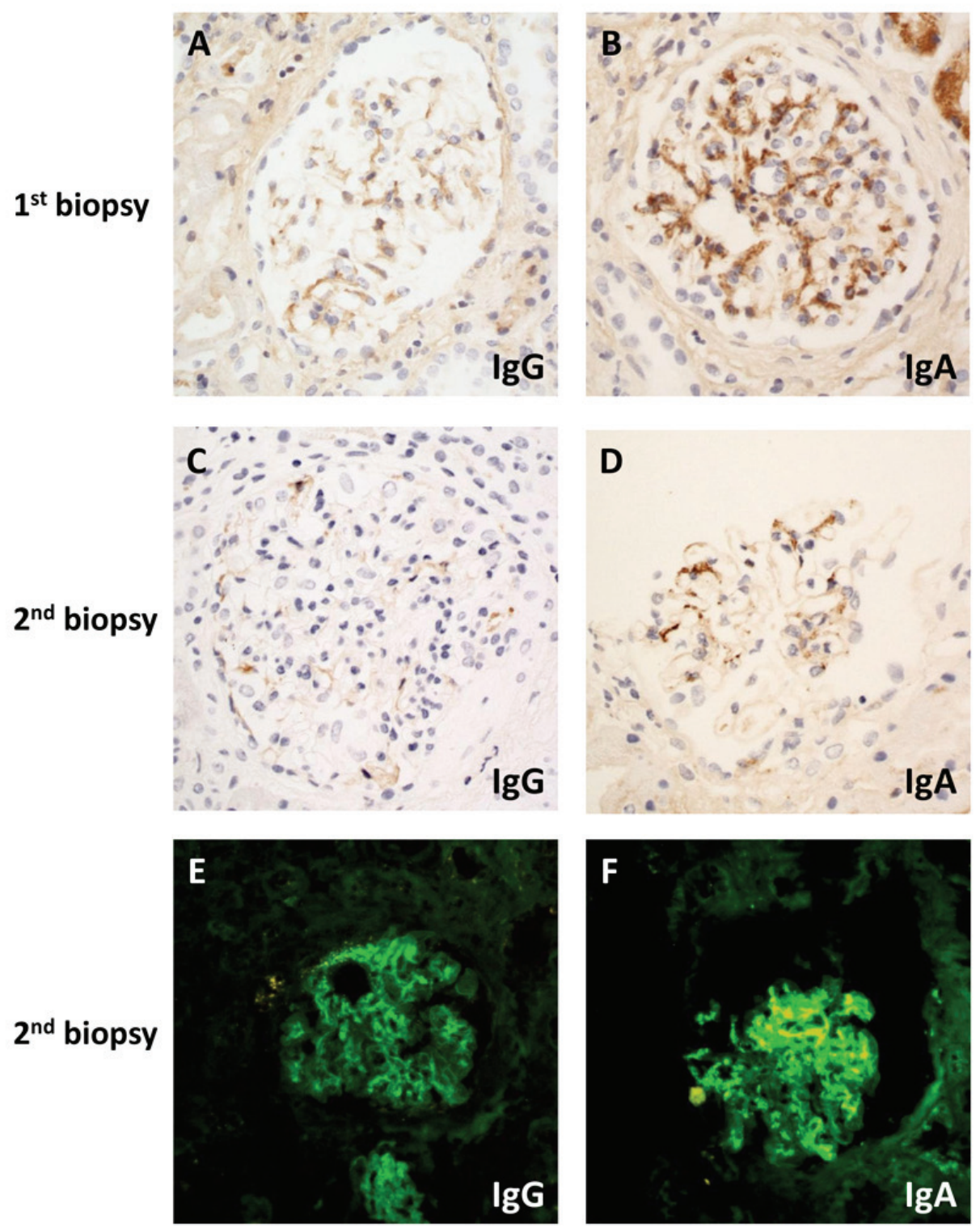

Figure 1. Microscopy of immunostaining on first (A, B) and second (C-F) biopsy by immunohistochemistry (A-D) or direct immunofluorescence (E, F): (A) weak granular mesangial staining for lgG; (B) moderate intensity mesangial staining for IgA; (C) absent IgG; (D) weak mesangial IgA staining; $(E)$ weak linear IgG; $(F)$ strong granular mesangial IgA staining. Original magnification $(A-F) \times 400$.

and urine electrophoresis were all unremarkable. Chest CT scan through his general practitioner showed no pulmonary hemorrhage.

A renal biopsy was performed on the second day of admission. Prior to the biopsy results, his urgent anti-GBM antibody titer by enzyme-linked immunosorbent assay (ELISA) (ORG 550, Orgentec Diagnostika, Matnz, Germany) became available and was moderately elevated at 57 units $/ \mathrm{mL}$ $(<20)$. Given the significant decline in renal function, hematuria and positive anti-GBM antibody, the presumptive diagnosis was RPGN secondary to anti-GBM disease, and urgent treatment was commenced before confirmation by histological diagnosis. This consisted of intravenous methylprednisolone, oral cyclophosphamide and urgent plasmapheresis with fresh frozen plasma as replacement fluid. Proton pump inhibitor, vitamin D and sulfamethoxazole/trimethoprim were also initiated as prophylaxis.

Light microscopy of the renal biopsy showed only 10 glomeruli, one of which was globally sclerosed. Only two glomeruli (20\%) were affected by cellular crescents and segmental necrosis, one of which showed mesangial proliferative changes. The rest of the glomeruli appeared normal. 

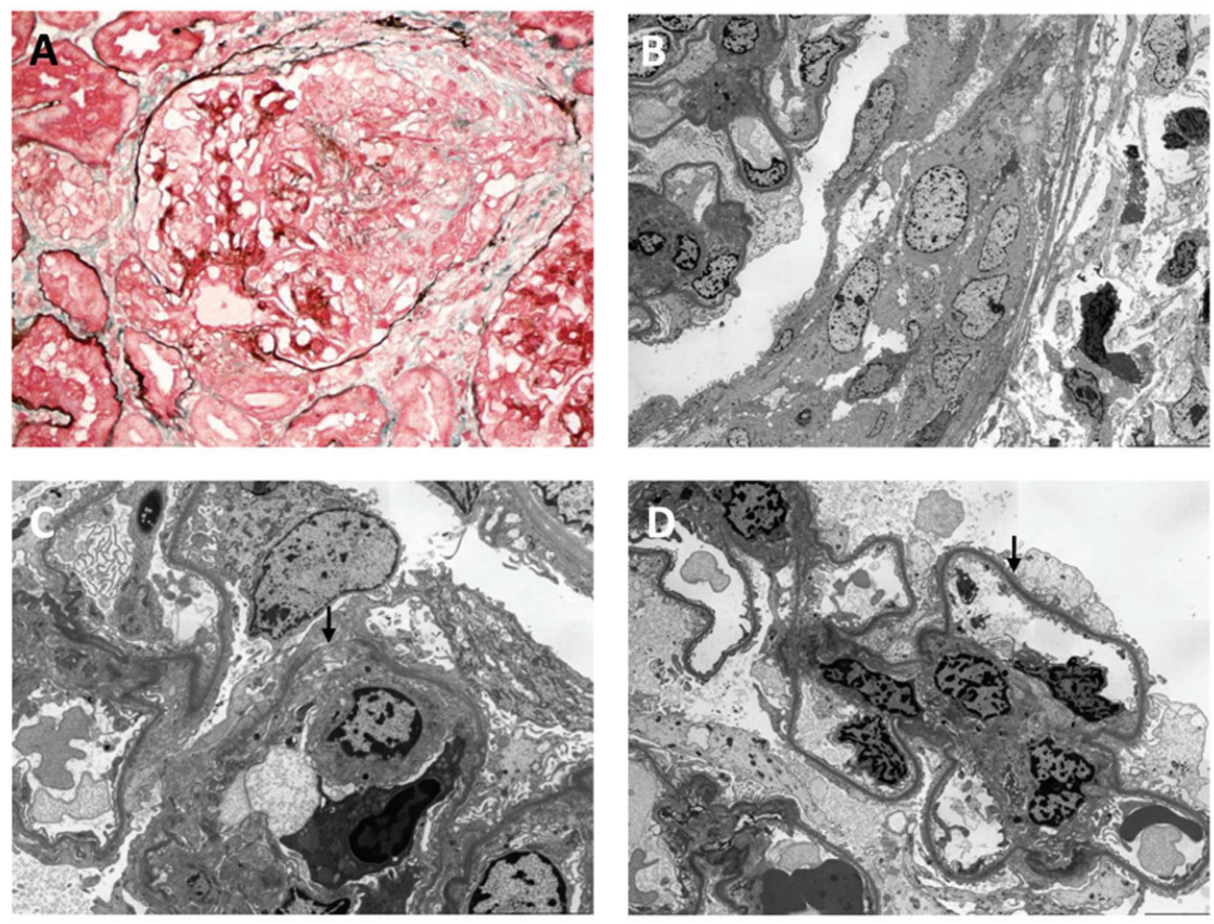

Figure 2. Light (A) and electron (B-D) microscopic findings on second biopsy: (A) segmental necrotizing lesion and cellular crescent (silver masson trichrome stain); (B) cellular crescent ( $\times 1,500)$; (C) defect in glomerular basement membrane (arrowed) $(\times$ 4,000); (D) foot process effacement (arrowed) $(\times 2,500)$. Original magnification $(A) \times 400 ;(B) \times 1,500 ;(C) \times 4,000 ;(D) \times 2,500$.

There was no significant interstitial fibrosis or tubular atrophy. IHC studies were performed instead of IF because of inadequate sampling. IHC staining showed moderate granu$\operatorname{lar}$ IgA (Fig. 1B) and weak granular IgG mesangial deposits without observable linear staining (Fig. 1A). The histological diagnosis therefore favored IgA nephropathy associated with crescent formation. Given the results of the biopsy and accompanying malfunction of the temporary vascular access, plasmapheresis was ceased after two sessions.

Two days after the renal biopsy, his kidney function worsened with serum creatinine rising to $2.81 \mathrm{mg} / \mathrm{dL}$. Due to the presence of circulating anti-GBM antibody and the absence of IF studies in the setting of segmental necrosis on the biopsy, there remained a strong suspicion of anti-GBM disease. A second renal biopsy was therefore performed 5 days after the initial biopsy. Light microscopy revealed a larger sample of 22 glomeruli, three of which were globally sclerosed. Crescents were identified in nine glomeruli $(41 \%)$, including a mixture of cellular, fibrocellular and occasional fibrous crescents, the latter associated with segmental necrosis (Fig. 2A). Mesangial hypercellularity was again focal and mild. Repeat IHC studies identified no IgG deposits (Fig. 1C) and weak mesangial IgA staining (Fig. 1D). IF studies revealed moderate IgG staining of the capillary loops in some glomeruli without mesangial staining (Fig. 1E) and marked granular mesangial IgA staining (Fig. 1F). Electron microscopy (EM) confirmed crescent formation (Fig. 2B) and localized GBM thinning and rupture (Fig. 2C). In the two glomeruli examined, there was no mesangial hypercellularity, no immune complex deposition, and variable foot process effacement (Fig. 2D). These findings were more consistent with crescentic and necrotising glomerulonephritis due to anti-GBM disease with co-existing mild IgA nephropathy.

Given the findings on the repeat renal biopsy, plasmapheresis was reinstituted with a total of nine treatments over a 16-day period, which was ceased 4 days after the antiGBM titer reduced below the limit of detection by ELISA. The patient remained on cyclophosphamide for almost 3 months with a cumulative dose of $11 \mathrm{~g}$ and was switched to azathioprine, which was ceased due to intolerance. At 7 months, his prednisolone dose was reduced to $5 \mathrm{mg}$, and his anti-GBM titers have remained undetectable when measured every 4 weeks. Kidney function has normalized with a serum creatinine of $1.12 \mathrm{mg} / \mathrm{dL}$ without proteinuria or microscopic hematuria.

\section{Discussion}

This case illustrates an atypical presentation of anti-GBM disease with associated initial diagnostic uncertainty. A systemic prodrome in the absence of infections or significantly impaired renal function is considered uncommon and/or less pronounced in anti-GBM disease, unless associated with 
concurrent ANCA-associated vasculitis [3]. The deterioration in kidney function is also usually more rapid than occurred in this case.

More significantly, the first renal biopsy indicated negative linear IgG IHC staining (positive linear IgG staining considered the hallmark of anti-GBM disease) and few crescents, findings which were further confounded by co-existing mild IgA nephropathy. It is well recognized that antiGBM disease may co-exist with other disease entities. IgA nephropathy [4], ANCA-associated vasculitis [5], membranous nephropathy [2], and immune complex glomerulonephritis [6] have all been described, and each has the potential to confound the diagnosis. In this case, IgA nephropathy was mild with only minor and focal mesangial proliferation. That it was not observed on EM was possibly due to the focal nature of the disease and sampling error. Rapidly progressive IgA nephropathy with crescent formation has been well described [7], and this was initially considered the likely histological diagnosis, although a strong suspicion of anti-GBM disease remained. First, segmental necrosis is not usually a feature of IgA nephropathy. Second, detection of anti-GBM antibody by ELISA has a high specificity ( $90.9 \%$ to $100.0 \%)$ [8], particularly for those assays using purified alpha-3(IV) antigen substrates. In this case, the clinical picture made a false positive test very unlikely. Finally, the lower sensitivity of IHC studies compared with IF could have missed the hallmark linear $\mathrm{IgG}$ deposits.

Our case of anti-GBM disease showed positive linear IgG staining by IF (despite being only moderate and focal) but negative by IHC methods on renal biopsy. The IgA staining also appeared more prominent by IF compared with IHC staining on the second biopsy. Consistent with previous reports, IF appears to be a more sensitive method for detecting linear $\operatorname{IgG}$ deposits $[9,10]$. It may therefore be the preferred diagnostic test when anti-GBM disease is suspected, although using antigen retrieval with microwave and pressure cooking may increase the sensitivity of IHC [11]. IF offers more rapid results on freshly frozen tissue sections while IHC can be performed with less tissue. IHC also has the double advantage of using paraffin-embedded formalinfixed tissue that can be stored long-term, and the immunostaining is permanent, in contrast to the limited lifespan of the IF signal. IF on paraffin sections, when compared to IF on fresh frozen sections, remains poor for the detection of linear IgG staining [12], and therefore attempts of IF to be performed on the paraffin sections from our first biopsy was unlikely to yield improve the sensitivity. In practice, the choice between IF and IHC largely depends on local expertise.

Another possible reason the initial IHC staining was negative is that the case may reflect a milder and/or earlier spectrum of anti-GBM disease. The deterioration in kidney function was less rapid and less marked than is often seen, and the serum creatinine was never high enough to consider dialysis. Furthermore, the anti-GBM titer was also only moderately elevated, becoming undetectable within 2 weeks of interrupted plasmapheresis, and the linear IgG deposits by IF were neither global nor did they affect all the examined glomeruli. Finally, crescents were neither prominent nor extensive on the first renal biopsy, the interpretation of which was limited by the small sample size.

Confirming the diagnosis by an early second renal biopsy, supported by the IF findings, was crucial in initiating prompt treatment and subsequent normalization of kidney function. Although the combination of plasmapheresis, cyclophosphamide and corticosteroid has revolutionized the outcome of the disease [13], the renal outcome remains heavily dependent on the renal function at presentation. Those with an initial serum creatinine $<5.7 \mathrm{mg} / \mathrm{dL}$ have a 5 -year renal survival $>90 \%$, while those dialysis-dependent at presentation have $<15 \%$ renal survival at 5 years [14]. Unfortunately, $>70 \%$ of patients present late with serum creatinine $>5.7 \mathrm{mg} / \mathrm{dL}$ and therefore poor renal outcomes [14]. Another smaller case series, however, reported that $36 \%$ of patients had normal kidney function on presentation, and all achieved favorable outcomes [15]. The milder form of antiGBM disease on presentation may be more common than previously thought, and it is therefore crucial to have a high index of suspicion for early diagnosis and treatment before the disease progresses.

In conclusion, our case study illustrates the importance of using clinical as well as pathological criteria in the atypical presentation of anti-GBM disease. Features such as a significant prodrome, a slower than usual decline in kidney function and less extensive crescent formation should not discount the diagnosis, and recognizing that other forms of glomerulonephritis may co-exist is essential. Linear IgG deposits on renal biopsy, the hallmark of anti-GBM disease, may not always be evident by IHC methods, and IF may be both more sensitive and provide a more rapid diagnosis. The presence of circulating anti-GBM antibody, in the setting of acute kidney injury and active urinary sediment, should prompt initiation and continuation of treatment for anti-GBM disease until it can be definitively excluded histologically. Finally, anti-GBM disease should be strongly suspected in any renal biopsy showing segmental necrosis associated with crescent formation and circulating anti-GBM antibody. Early recognition and initiation of treatment are paramount for favorable outcomes.

\section{Financial Disclosure}

None.

\section{References}

1. Saus J, Wieslander J, Langeveld JP, Quinones S, Hud- 
son BG. Identification of the Goodpasture antigen as the alpha 3(IV) chain of collagen IV. J Biol Chem. 1988;263(26):13374-13380.

2. Pusey CD. Anti-glomerular basement membrane disease. Kidney Int. 2003;64(4):1535-1550.

3. Kluth DC, Rees AJ. Anti-glomerular basement membrane disease. J Am Soc Nephrol. 1999;10(11):24462453.

4. Gao B, Li M, Xia W, Wen Y, Qu Z. Rapidly progressive glomerulonephritis due to anti-glomerular basement membrane disease accompanied by $\operatorname{IgA}$ nephropathy: a case report. Clin Nephrol. 2014;81(2):138-141.

5. Jayne DR, Marshall PD, Jones SJ, Lockwood CM. Autoantibodies to GBM and neutrophil cytoplasm in rapidly progressive glomerulonephritis. Kidney Int. 1990;37(3):965-970.

6. Cui Z, Zhao MH, Wang SX, Liu G, Zou WZ, Wang HY. Concurrent antiglomerular basement membrane disease and immune complex glomerulonephritis. Ren Fail. 2006;28(1):7-14.

7. Jennette JC. Rapidly progressive crescentic glomerulonephritis. Kidney Int. 2003;63(3):1164-1177.

8. Sinico RA, Radice A, Corace C, Sabadini E, Bollini B. Anti-glomerular basement membrane antibodies in the diagnosis of Goodpasture syndrome: a comparison of different assays. Nephrol Dial Transplant. 2006;21(2):397-401.

9. Howat AJ, Armour A, Ellis IO. Microinvasive lobular carcinoma of the breast. Histopathology. 2000;37(5):477478.

10. Molne J, Breimer ME, Svalander CT. Immunoperoxidase versus immunofluorescence in the assessment of human renal biopsies. Am J Kidney Dis. 2005;45(4):674-683.

11. Shi S, Zhang P, Cheng Q, Wu J, Cui J, Zheng Y, Bai $\mathrm{XY}$, et al. Immunohistochemistry of deparaffinised sections using antigen retrieval with microwave combined pressure cooking versus immunofluorescence in the assessment of human renal biopsies. J Clin Pathol. 2013;66(5):374-380.

12. Nasr SH, Galgano SJ, Markowitz GS, Stokes MB, D'Agati VD. Immunofluorescence on pronase-digested paraffin sections: a valuable salvage technique for renal biopsies. Kidney Int. 2006;70(12):2148-2151.

13. Lockwood CM, Rees AJ, Pearson TA, Evans DJ, Peters DK, Wilson CB. Immunosuppression and plasmaexchange in the treatment of Goodpasture's syndrome. Lancet. 1976;1(7962):711-715.

14. Levy JB, Turner AN, Rees AJ, Pusey CD. Long-term outcome of anti-glomerular basement membrane antibody disease treated with plasma exchange and immunosuppression. Ann Intern Med. 2001;134(11):10331042.

15. Ang C, Savige J, Dawborn J, Miach P, Heale W, Clarke $\mathrm{B}$, Sinclair RS. Anti-glomerular basement membrane (GBM)-antibody-mediated disease with normal renal function. Nephrol Dial Transplant. 1998;13(4):935-939. 